\title{
联合线性判别和图正则的任务导向型跨模态检索
}

\author{
代瑾，陈莹* \\ (江南大学轻工过程先进控制教育部重点实验室 无锡 214122) \\ (chenying@jiangnan.edu.cn)
}

\begin{abstract}
摘 要：针对现有的基于公共子空间的跨模态检索方法对不同检索任务的差异性、检索模态的语义一致性考虑不足 的问题，提出一种联合线性判别和图正则的任务导向型跨模态检索方法. 该方法在一个联合学习框架中为不同的检 索任务构建不同的映射机制，将不同模态的数据映射到公共子空间中以进行相似性度量; 学习过程中结合相关性分 析和单模态语义回归，保留成对数据间的相关性以及增强查询模态样本的语义准确性，同时利用线性判别分析保证 检索模态样本的语义一致性; 还为不同模态的数据构建局部近邻图以保留结构信息, 从而提升跨模态检索的性能. 在 Wikipedia 和 Pascal Sentence 这 2 个跨模态数据集上的实验结果表明, 该方法在不同检索任务上的平均 mAP 值比 12 种现有方法分别提升了 $1.0 \% \sim 16.0 \%$ 和 $1.2 \% \sim 14.0 \%$.
\end{abstract}

关键词：跨模态检索；线性判别分析；图正则化；任务导向

中图法分类号: TP391.41 DOI: 10.3724/SP.J.1089.2021.18345

\section{Joint Linear Discrimination and Graph Regularization for Task-Oriented Cross- Modal Retrieval}

\author{
Dai Jin and Chen Ying ${ }^{*}$ \\ (Key Laboratory of Advanced Process Control for Light Industry (Ministry of Education), Jiangnan University, Wuxi 214122)
}

\begin{abstract}
Aiming at the problem of insufficient consideration of the differences between different retrieval tasks and semantic consistency of retrieval-modal data in the current common subspace based cross-modal retrieval algorithms, a task-oriented cross-modal retrieval based on jointing linear discrimination and graph regularization is proposed. The approach constructed different mapping mechanisms for retrieval tasks in a joint learning framework, and mapped multi-modal data into common subspaces for similarity measuring. During the learning process, correlation analysis and single-modal semantic regression were combined to preserve the correlation between paired data and enhance the semantic accuracy of query-modal data. Simultaneously, linear discrimination analysis was utilized to ensure semantic consistency of retrieval-modal samples. The approach also constructed local neighbor graphs for multi-modal data to preserve structural information, which can improve the retrieval performance. Experiments results on two cross-modal datasets, namely Wikipedia and Pascal Sentence showed that the average mAP value on different retrieval tasks of the proposed method had respectively increased by $1.0 \%-16.0 \%$ and $1.2 \%-14.0 \%$ compared with the twelve existing methods.
\end{abstract}

Key words: cross-modal retrieval; linear discriminant analysis; graph regularization; task-oriented

收稿日期: 2020-05-05; 修回日期: 2020-09-21. 基金项目：国家自然科学基金(61573168). 代瑾(1996一), 女, 硕士研究生, 主要 研究方向为计算机视觉; 陈莹(1976-), 女, 博士, 教授, 博士生导师, CCF 会员, 论文通讯作者, 主要研究方向为计算机视觉、信息 融合. 
移动互联网时代，信息数据如图片、文本、音 视频等呈现爆炸式增长. 如何从这些语义关联且 模态多样的数据中获取有价值的信息显得尤为重 要, 跨模态检索也因此备受关注. 与图片检索等单 模态检索不同，跨模态检索旨在实现跨越不同模 态的信息检索，即用户提交任意查询模态的样本， 可检索到与之相关的其他模态的样本，而单模态 检索只能检索到与查询样本模态相同的结果. 因 此，跨模态检索更能满足用户日益增长的检索需 求. 而不同模态的数据因结构分布、特征表示等不 同存在异构鸿沟问题, 如何挖掘其匹配关系, 缩短 其异构差距，提升检索准确率，是跨模态检索叒待 解决的问题 ${ }^{[1]}$.

如何度量异构特征表示间的相似性，实现跨 模态检索, 常用的解决方法是为多模态数据构建 公共子空间，将其映射到子空间中学习统一表征， 再利用简单的距离度量方法如欧几里得度量等度 量其相似性. 早期的一些代表性工作有典型相关

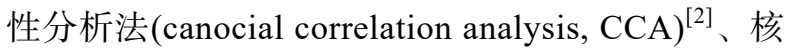
典型相关性分析法 (kernel canonical correlation analysis, KCCA) ${ }^{[3]}$ 、偏最小二乘法 (partial least squares, PLS) ${ }^{[4]}$ 、跨模态因子分析法 (cross-modal factor analysis, CFA $)^{[5]}$ 等，通过典型变量等方式最 大化不同模态成对数据间的相关性习得一组映射 矩阵, 将其转化到同维子空间. 为了学习更具语义 判别性的子空间, Rasiwasia 等 ${ }^{[6]}$ 利用 CCA 学习多 模态数据相关性的同时通过多类逻辑回归加人语 义标签信息，以提升检索性能. Gong 等 ${ }^{[7]}$ 利用语义 信息将 CCA 扩展到多视角. Zhai 等 ${ }^{[8]}$ 提出为多模态 数据构建图模型来共同挖掘语义信息和相关信息. Zhang 等 ${ }^{[9]}$ 采用标签图约束项保留不同模态样本间 固有的语义一致结构. Qi 等 ${ }^{[10]}$ 提出基于线性判别 分析(cross-media retrieval based on linear discriminant analysis, CRLDA)的方法，其在进行语义回归 时通过线性判别分析项保留文本模态的强判别性, 并利用相关性分析将其迁移到相应的视觉特征上. 然而，这些方法忽略了不同检索任务的差异性，只 学习一组线性(非线性)映射, 使得每个检索任务的 性能无法达到各自最优.

根据不同检索任务的特性, Wei 等 ${ }^{[1]}$ 提出模态 依赖的跨模态检索 (modality dependent cross-media retrieval, MDCR)方法, 首次为各检索任务学习不 同的映射机制，通过单模态语义回归增强查询模 态样本的语义准确性, 从而在各检索任务中获得 更好的性能. 近几年, 在此基础上相继提出一些工
作 ${ }^{[2-14]}$. 其中, $Y$ 等 $^{[12]}$ 在 MDCR 的基础上, 针对不 同类别的样本学习自适应的映射矩阵, 充分挖掘 不同类之间的语义重叠部分的潜在共享信息. 然 而, 这些方法都只考虑了增强查询模态的语义准 确性，忽略了保留检索模态的语义一致性.

针对以上方法存在的问题，考虑通过线性判 别分析可以对数据进行有监督聚类, 本文在文献 [10]的基础上提出联合线性判别和图正则的任务 导向型跨模态检索方法. 不同于以往的任务导向 型方法只保留查询模态的语义一致性, 本文方法 学习任务特定的映射矩阵时利用判别性分析项在 不干扰查询模态语义准确性的同时尽可能保留了 检索模态的语义一致性, 还为多模态数据构建了 图模型, 用于挖掘各模态内部样本间的局部近邻 结构信息，以进一步优化该检索框架.

\section{1 模态依赖的跨模态检索方法及问题分析}

MDCR 方法为图片检索文本(Image2Text, I2T) 和文本检索图片(Text2Image, T2I)任务学习了 2 组 映射矩阵 $\boldsymbol{V} \in \mathbb{R}^{c \times p}$ 和 $\boldsymbol{W} \in \mathbb{R}^{c \times q}$, 但不同检索任务 中目标函数的整体框架一致, 都结合了图文模态 成对样本间的相关性和查询模态样本对其语义标 签向量的回归，总优化框架为

$$
\min _{\boldsymbol{V}, \boldsymbol{W}} f(\boldsymbol{V}, \boldsymbol{W})=C(\boldsymbol{V}, \boldsymbol{W})+L(\boldsymbol{V}, \boldsymbol{W})+R(\boldsymbol{V}, \boldsymbol{W})
$$

其中, $C(\boldsymbol{V}, \boldsymbol{W})$ 为不同模态数据间的相关性分析 项; $L(\boldsymbol{V}, \boldsymbol{W})$ 为各检索任务中查询模态样本的语义 回归项; $R(\boldsymbol{V}, \boldsymbol{W})$ 为图片和文本特征的正则化项. 不同检索任务中只有语义回归项不同, I2T 任务只 考虑图片模态的语义标签信息, 其语义回归项为

$$
\boldsymbol{L}\left(\boldsymbol{V}_{1}, \boldsymbol{W}_{1}\right)=\left\|\boldsymbol{X} \boldsymbol{V}_{1}^{\mathrm{T}}-\boldsymbol{S}\right\|_{\mathrm{F}}^{2}
$$

T2I 任务只考虑文本模态的语义标签信息，其语义 回归项为

$$
\boldsymbol{L}\left(\boldsymbol{V}_{2}, \boldsymbol{W}_{2}\right)=\left\|\boldsymbol{T} \boldsymbol{W}_{2}^{\mathrm{T}}-\boldsymbol{S}\right\|_{\mathrm{F}}^{2}
$$

其中, $\boldsymbol{X}=\left[\boldsymbol{x}_{1}, \cdots, \boldsymbol{x}_{n}\right]^{\mathrm{T}} \in \mathbb{R}^{n \times p}$ 为训练图片的特征矩 阵; $\boldsymbol{T}=\left[\boldsymbol{t}_{1}, \cdots, \boldsymbol{t}_{n}\right]^{\mathrm{T}} \in \mathbb{R}^{n \times q}$ 为训练文本的特征矩阵; $\boldsymbol{V}_{1} \in \mathbb{R}^{c \times p}, \boldsymbol{W}_{1} \in \mathbb{R}^{c \times q}$ 为 $\mathrm{I} 2 \mathrm{~T}$ 任务习得的一组映射矩 阵, 分别对应图片、文本特征; 相应地, $\boldsymbol{V}_{2} \in \mathbb{R}^{c \times p}, \boldsymbol{W}_{2} \in \mathbb{R}^{c \times q}$ 为 $\mathrm{T} 2 \mathrm{I}$ 任务习得的一组映射 矩阵. 
MDCR 方法通过式(2)(3)中的单模态语义回归 来增强各检索任务中查询模态样本的语义准确性. 以图片检索文本为例，对该任务而言，图片在同维 子空间中的特征表示优劣比文本在同维子空间中 的特征表示优劣更重要, 如果图片的语义特征预 测错误，则很难再检索到与该图片语义一致的文 本. 而数据集中与图片成对的文本描述常包含与 图片内容无关的语义信息, 如图 1 所示 Wikipedia 数据集中成对的图片和文本, 左半图和右半文均 属于“sport”类别. 其中, 从图 1 的左半图可判断出
这是一场冰球比赛，而右半文不是对该图片的简 单解释，如“一群穿着球衣的运动员在雪白的赛场 上争抢冰球”, 而是介绍左半图中该比赛背后的争 议以及奥林匹克历史上对专业运动员排斥的历史. 显然，这种无关描述会干扰图片对其类别标签的 回归，导致其预测错误. 然而完全舍弃文本模态的 语义信息会导致语义类别一致的文本映射到子空 间后无法聚集，从而影响检索精度. 因此，在不干 扰查询模态样本语义准确性的情况下保留检索模 态的语义一致性是十分重要的.

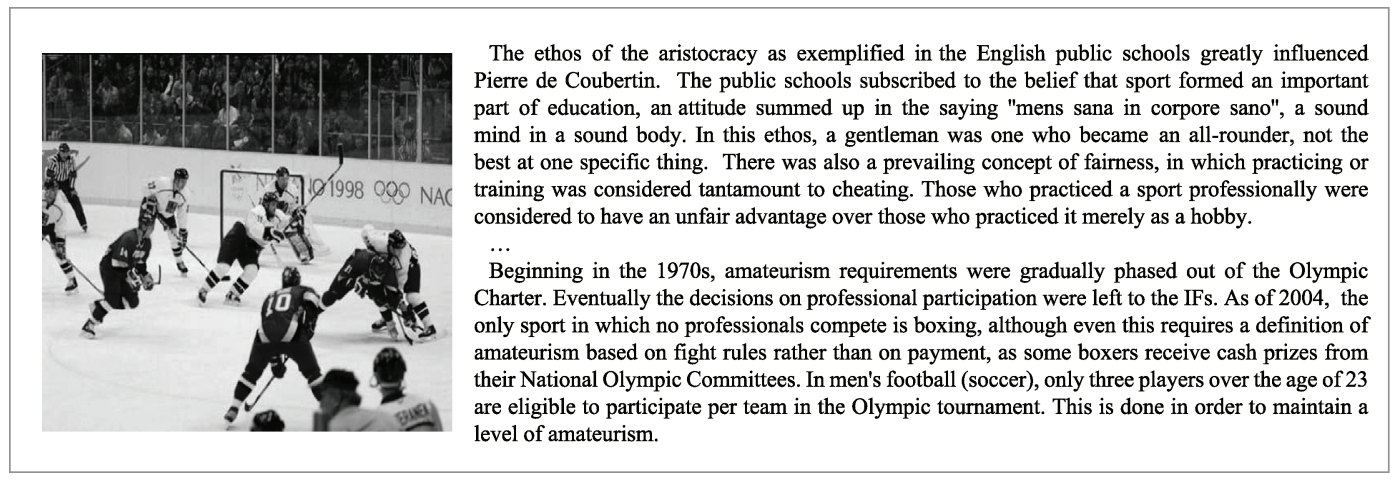

图 1 Wikipedia 数据集中的图片文本对示例

考虑线性判别分析可以对数据进行有监督聚 类，本文提出对不同检索任务中的检索模态进行 线性判别分析，通过减小其类内差距、增大其类间 差距使检索模态语义一致的样本映射到同维子空 间后聚集在一起. 那么, 当查询图片(文本)的语义 预测正确时，就可以密集地检索到相关的文本(图 片), 从而提升跨模态检索的性能.

\section{2 本文方法}

\section{1 概 述}

本文方法是基于图片和文本之间的跨模态检 索, 整体框架如图 2 所示. 其中, 圆圈(方块)表示 图片(文本), 实心(空心)图案表示已(未)标记数据.

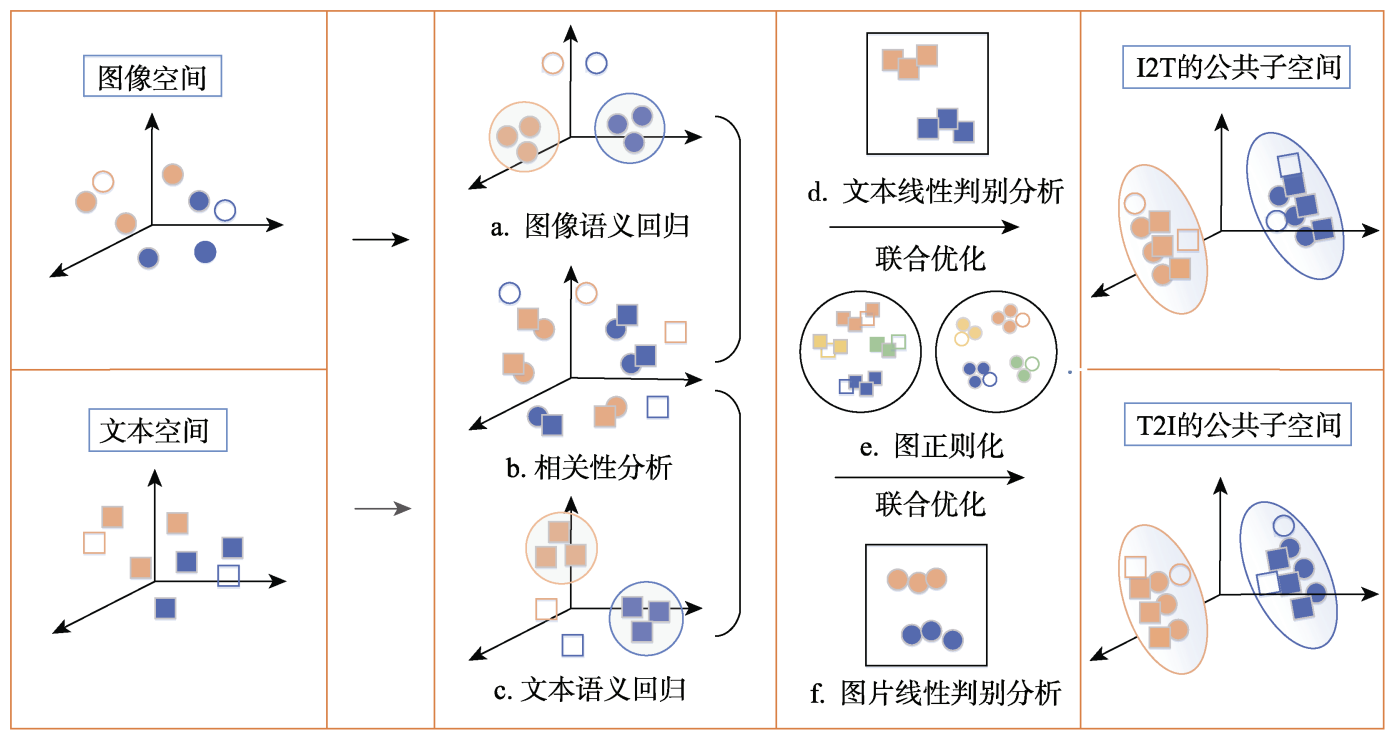

图 2 本文方法框架图 
考虑不同检索任务的特性, 该方法为 $\mathrm{I} 2 \mathrm{~T}$ 和 T2I 任务学习不同的映射机制. 图 2a 和图 2c 分别为 $\mathrm{I} 2 \mathrm{~T}$ 和 T2I 任务中查询模态样本(图片或文本)的单模 态语义回归，保证其映射到公共子空间后语义和类 别是一致的. 图 $2 \mathrm{~b}$ 为图片和文本间的相关性分析, 通过该项约束成对的图片和文本在公共子空间中的 位置仍相近. 图 2d 和图 2f 分别为 I2T 和 T2I 任务中 检索模态样本(文本或图片)的线性判别分析项, 通 过该项使检索模态样本类内靠近、类间远离，保证 检索模态的语义一致性. 图 2e 为图片和文本的综 合图正则化项, 利用已标记和未标记数据为图片 和文本构建其模态内的 $k$ 最近邻 $(k$-nearest neighbor, $k \mathrm{NN}$ ) 局部结构图, 通过该项使图片和文 本数据映射到子空间后都尽可能保留其在原始特征 空间中的近邻分布. 最后在一个统一框架下进行优 化求解, 联合图 $2 a$ 、图 $2 b$ 、图 $2 d$ 和图 $2 \mathrm{e}$ 学习一组 映射矩阵并用于 $\mathrm{I} 2 \mathrm{~T}$ 任务, 联合图 2c、图 $2 \mathrm{~b}$ 、图 $2 \mathrm{f}$ 和图 $2 \mathrm{e}$ 学习一组映射矩阵并用于 T2I 任务.

下面介绍本文的形式化定义. 假设已标记多模 态数据集为 $\boldsymbol{D}=\left\{\boldsymbol{D}^{i}, \boldsymbol{D}^{t}\right\}$, 其中 $i, t$ 分别表示图片 模态和文本模态. 已标记图片数据集 $D^{i}=\left\{\boldsymbol{i}_{p}, \boldsymbol{s}_{p}\right\}_{p=1}^{n}$ 中 $\boldsymbol{i}_{p} \in \mathbb{R}^{d^{i}}$ 是第 $p$ 张图片且具有相应的标签 $\boldsymbol{s}_{p}$, 已标记文本数据集 $\boldsymbol{D}^{t}=\left\{\boldsymbol{t}_{p}, \boldsymbol{s}_{p}\right\}_{p=1}^{n}$ 中 $\boldsymbol{t}_{p} \in \mathbb{R}^{d^{t}}$ 是第 $p$ 个文本且具有相应的标签 $\boldsymbol{s}_{p}, n$ 为已标记样本总 数. 未标记多模态数据集为 $\boldsymbol{D}^{*}=\left\{\boldsymbol{D}^{i^{*}}, \boldsymbol{D}^{t^{*}}\right\}$, 其中图 片模态为 $\boldsymbol{D}^{i^{*}}=\left\{\boldsymbol{i}_{p}\right\}_{p=n+1}^{n+m}$, 文本模态为 $\boldsymbol{D}^{t^{*}}=\left\{\boldsymbol{t}_{p}\right\}_{p=n+1}^{n+m}$, $m$ 为未标记样本总数. 所有图片的矩阵为 $\boldsymbol{I}^{*}=\left[\boldsymbol{i}_{1}, \boldsymbol{i}_{2}, \cdots, \boldsymbol{i}_{n+m}\right] \in \mathbb{R}^{(n+m) \times d^{i}}$, 所有文本的矩阵为 $\boldsymbol{T}^{*}=\left[\boldsymbol{t}_{1}, \boldsymbol{t}_{2}, \cdots, \boldsymbol{t}_{n+m}\right] \in \mathbb{R}^{(n+m) \times d^{t}}$. 其中, 已标记图片 矩阵为 $\boldsymbol{I}=\left[\boldsymbol{i}_{1}, \boldsymbol{i}_{2}, \cdots, \boldsymbol{i}_{n}\right] \in \mathbb{R}^{n \times d^{i}}$, 已标记文本矩阵为 $\boldsymbol{T}=\left[\boldsymbol{t}_{1}, \boldsymbol{t}_{2}, \cdots, \boldsymbol{t}_{n}\right] \in \mathbb{R}^{n \times d^{t}}$ ，其相应的标签矩阵为 $\boldsymbol{S}=\left\{\boldsymbol{s}_{1}, \boldsymbol{s}_{2}, \cdots, \boldsymbol{s}_{n}\right\} \in \mathbb{R}^{n \times c}, c$ 为数据集的语义类别数.

\section{2 目标函数}

本文方法旨在为图片和文本学习 2 组映射矩 阵 $\boldsymbol{X} \in \mathbb{R}^{c \times d^{i}}$ 和 $\boldsymbol{Y} \in \mathbb{R}^{c \times d^{t}}$, 将其映射到可度量相似 度的公共子空间，以进行跨模态检索. 其通用框架 定义为

$$
\begin{aligned}
& \underset{\boldsymbol{X}, \boldsymbol{Y}}{\arg \min } f(\boldsymbol{X}, \boldsymbol{Y})=C(\boldsymbol{X}, \boldsymbol{Y})+L(\boldsymbol{X}, \boldsymbol{Y})+R(\boldsymbol{X}, \boldsymbol{Y})+ \\
& D(\boldsymbol{X}, \boldsymbol{Y})+G(\boldsymbol{X}, \boldsymbol{Y})
\end{aligned}
$$

由式(4)可知, 该方法的目标函数由 5 项构成, 包括 相关性分析项 $C(\boldsymbol{X}, \boldsymbol{Y})$ 、语义回归项 $L(\boldsymbol{X}, \boldsymbol{Y})$ 、正则 化项 $R(\boldsymbol{X}, \boldsymbol{Y})$ 、线性判别分析项 $D(\boldsymbol{X}, \boldsymbol{Y})$ 以及图正则 化项 $G(\boldsymbol{X}, \boldsymbol{Y})$. 下面详细介绍各部分组成和作用.

\subsection{1 相关性分析项}

$C(\boldsymbol{X}, \boldsymbol{Y})$ 表示子空间中不同模态成对数据间 的距离, 最小化该项可最大化成对数据间的相关 性, 保证成对的多模态数据在公共子空间中的位 置相近，该项可表示为如下约束 ${ }^{[15]}$

$$
C(\boldsymbol{X}, \boldsymbol{Y})=\lambda\left\|\boldsymbol{I} \boldsymbol{X}^{\mathrm{T}}-\boldsymbol{T} \boldsymbol{Y}^{\mathrm{T}}\right\|_{\mathrm{F}}^{2}
$$

其中, $\|\cdot\|_{\mathrm{F}}$ 表示矩阵的 $\mathrm{F}$ 范数.

\section{2 .2 语义回归项}

$L(\boldsymbol{X}, \boldsymbol{Y})$ 代表不同检索任务中查询模态样本 的原始特征向量向其相应的语义特征向量的回归, 通过该项可使子空间中语义相同的同模态数据聚 集在一起. 因此 I2T 任务中, 语义回归项为

$$
L(\boldsymbol{X}, \boldsymbol{Y})=(1-\lambda)\left\|\boldsymbol{I} \boldsymbol{X}^{\mathrm{T}}-\boldsymbol{S}\right\|_{\mathrm{F}}^{2}
$$

$\mathrm{T} 2 \mathrm{I}$ 任务中, 语义回归项定义为

$$
L(\boldsymbol{X}, \boldsymbol{Y})=(1-\lambda)\left\|\boldsymbol{T} \boldsymbol{Y}^{\mathrm{T}}-\boldsymbol{S}\right\|_{\mathrm{F}}^{2}
$$

\subsection{3 正则化项}

$R(\boldsymbol{X}, \boldsymbol{Y})$ 主要用于控制映射矩阵 $\boldsymbol{X}$ 和 $\boldsymbol{Y}$ 的复 杂性，避免过拟合. 其定义为

$$
R(\boldsymbol{X}, \boldsymbol{Y})=\alpha_{1}\|\boldsymbol{X}\|_{\mathrm{F}}^{2}+\alpha_{2}\|\boldsymbol{Y}\|_{\mathrm{F}}^{2}
$$

\subsection{4 线性判别分析项}

仅关注查询模态的语义信息是不够的，合理 地利用检索模态的语义信息有助于提升检索性能. 本文通过线性判别分析项使检索模态类内样本尽 可能靠近、类间样本尽可能远离, 在不干扰查询模 态样本语义信息的情况下保留检索模态样本的语 义一致性. 与语义回归项类似, 检索任务不同, 线 性判别分析项也不同, 各检索任务中只保留检索 模态样本的线性判别分析项.

（1） I2T 任务中保留文本模态对应的线性判别 分析项. 假设文本训练特征的第 $j$ 类均值为 $\overline{\boldsymbol{m}}_{j}^{t}$, 所有文本训练特征的均值 $\overline{\boldsymbol{m}}^{t}, n_{j}$ 为每类样本的数 量, 那么文本训练特征的类内散度矩阵 $\boldsymbol{M}_{a}^{t}$ 以及类 间散度矩阵 $\boldsymbol{M}_{b}^{t}$ 分别为

$$
\boldsymbol{M}_{a}^{t}=\frac{1}{n} \sum_{j=1}^{c} \sum_{p=1 \& s_{p}=j}^{n}\left(\boldsymbol{t}_{p}-\overline{\boldsymbol{m}}_{j}^{t}\right)\left(\boldsymbol{t}_{p}-\overline{\boldsymbol{m}}_{j}^{t}\right)^{\mathrm{T}}
$$




$$
\boldsymbol{M}_{b}^{t}=\frac{1}{n} \sum_{j=1}^{c} n_{j}\left(\overline{\boldsymbol{m}}_{j}^{t}-\overline{\boldsymbol{m}}^{t}\right)\left(\overline{\boldsymbol{m}}_{j}^{t}-\overline{\boldsymbol{m}}^{t}\right)^{\mathrm{T}}
$$

由此可得，线性判别分析项的通用目标函数为

$$
\min _{\boldsymbol{Y} \boldsymbol{Y}^{\mathrm{T}}=\boldsymbol{I}_{C}} \frac{\operatorname{tr}\left(\boldsymbol{Y} \boldsymbol{M}_{a}^{t} \boldsymbol{Y}^{\mathrm{T}}\right)}{\operatorname{tr}\left(\boldsymbol{Y} \boldsymbol{M}_{b}^{t} \boldsymbol{Y}^{\mathrm{T}}\right)} .
$$

其中, $\boldsymbol{Y}$ 是文本模态对应的映射矩阵. 若要最小化 该项，那么最大化分母 $\operatorname{tr}\left(\boldsymbol{Y} \boldsymbol{M}_{b}^{t} \boldsymbol{Y}^{\mathrm{T}}\right)$ 的同时要最小 化分子 $\operatorname{tr}\left(\boldsymbol{Y} \boldsymbol{M}_{a}^{t} \boldsymbol{Y}^{\mathrm{T}}\right)$. 根据文献[16]，该项的优化问 题可转化为最小化边界的形式, 即 $\mathrm{I} 2 \mathrm{~T}$ 任务中对应 的线性判别分析项为

$$
D(\boldsymbol{X}, \boldsymbol{Y})=\beta\left(\operatorname{tr}\left(\boldsymbol{Y} \boldsymbol{M}_{a}^{t} \boldsymbol{Y}^{\mathrm{T}}\right)-\operatorname{tr}\left(\boldsymbol{Y} \boldsymbol{M}_{b}^{t} \boldsymbol{Y}^{\mathrm{T}}\right)\right)
$$

（2）T2I 任务中只保留图片模态的线性判别分 析项. 假设图片训练特征的第 $j$ 类均值为 $\overline{\boldsymbol{m}}_{j}^{i}$, 所 有图片训练特征的均值 $\overline{\boldsymbol{m}}^{i}, n_{j}$ 为每类样本的数 量, 那么图片训练特征的类内散度矩阵 $\boldsymbol{M}_{a}^{i}$ 以及类 间散度矩阵 $\boldsymbol{M}_{b}^{i}$ 分别为

$$
\begin{gathered}
\boldsymbol{M}_{a}^{i}=\frac{1}{n} \sum_{j=1}^{c} \sum_{p=1 \& \boldsymbol{s}_{p}=j}^{n}\left(\boldsymbol{i}_{p}-\overline{\boldsymbol{m}}_{j}^{i}\right)\left(\boldsymbol{i}_{p}-\overline{\boldsymbol{m}}_{j}^{i}\right)^{\mathrm{T}} \\
\boldsymbol{M}_{b}^{i}=\frac{1}{n} \sum_{j=1}^{c} n_{j}\left(\overline{\boldsymbol{m}}_{j}^{i}-\overline{\boldsymbol{m}}^{i}\right)\left(\overline{\boldsymbol{m}}_{j}^{i}-\overline{\boldsymbol{m}}^{i}\right)^{\mathrm{T}}
\end{gathered}
$$

可得 T2I 任务中对应的线性判别分析项为

$$
D(\boldsymbol{X}, \boldsymbol{Y})=\beta\left(\operatorname{tr}\left(\boldsymbol{X} \boldsymbol{M}_{a}^{i} \boldsymbol{X}^{\mathrm{T}}\right)-\operatorname{tr}\left(\boldsymbol{X} \boldsymbol{M}_{b}^{i} \boldsymbol{X}^{\mathrm{T}}\right)\right)
$$

\section{2 .5 图正则化项}

不同模态原始空间中的样本存在局部近邻关 系 ${ }^{[17]}$, 样本距离越近，特征分布信息更为一致，语 义更为相似，因而在公共空间中保持样本的原始 分布信息有助于同类聚集，从而提升检索准确率. 为了学习图片和文本的原始分布信息，可根据模 态内的局部近邻信息构建各自的模态图 ${ }^{[18]}$, 并利 用谱图理论定义图正则化项 $G(\boldsymbol{X}, \boldsymbol{Y})$, 从而尽可能 地使在原始空间中距离相近的样本映射到公共空 间后仍相近. 以图片模态为例, 定义一个无向图 $\boldsymbol{G}_{i}\left(\boldsymbol{V}_{i}, \boldsymbol{W}_{i}\right)$, 对称相似矩阵 $\boldsymbol{W}_{i}=\left\{w_{p q}\right\}_{(n+m) \cdot(n+m)}$, 其 中, $w_{p q}$ 表示数据集中第 $p$ 张图片和第 $q$ 张图片之 间的相似度，该项利用 $k$ 近邻定义为

$$
w_{p q}=\left\{\begin{array}{l}
1, \text { if } i_{p} \in N_{k}\left(i_{q}\right) \quad \text { or } \quad i_{p} \in N_{k}\left(i_{q}\right) \\
0, \text { otherwise }
\end{array}\right.
$$

其中, $N_{k}\left(i_{q}\right)$ 表示数据集中 $i_{q}$ 元素的 $k$ 近邻. 假设
所有图片投影到公共子空间中的表示为 $\boldsymbol{A}=\boldsymbol{I}^{*} \boldsymbol{X}^{\mathrm{T}}$, 那么图片模态的图正则化项为

$$
\begin{gathered}
g_{i}(\boldsymbol{X}, \boldsymbol{Y})=\frac{1}{2} \sum_{p, q=1}^{n+m}\left\|\frac{a_{p}}{\sqrt{d_{p p}}}-\frac{a_{q}}{\sqrt{d_{q q}}}\right\|^{2} w_{p q}= \\
\operatorname{tr}\left(\boldsymbol{X I}^{\mathrm{T}} \boldsymbol{L}_{i} \boldsymbol{I X}^{\mathrm{T}}\right)
\end{gathered}
$$

其中, $\boldsymbol{L}_{i}=\boldsymbol{I}-\boldsymbol{D}_{i}^{-1 / 2} \boldsymbol{W}_{i} \boldsymbol{D}_{i}^{-1 / 2}$ 是一对称半正定矩阵, 度矩阵 $\boldsymbol{D}_{i}$ 是一个对角矩阵, 其对角元素为 $d_{p p}=\sum_{q} w_{p q}$. 同样, 可以得到文本模态的图正则 化项为

$$
\begin{gathered}
g_{t}(\boldsymbol{X}, \boldsymbol{Y})=\frac{1}{2} \sum_{p, q=1}^{n+m}\left\|\frac{b_{p}}{\sqrt{d_{p p}}}-\frac{b_{q}}{\sqrt{d_{q q}}}\right\|^{2} w_{p q}= \\
\operatorname{tr}\left(\boldsymbol{Y} \boldsymbol{T}^{\mathrm{T}} \boldsymbol{L}_{t} \boldsymbol{T} \boldsymbol{Y}^{\mathrm{T}}\right)
\end{gathered}
$$

综上, 最终的图正则化项 $G(\boldsymbol{X}, \boldsymbol{Y})$ 定义为

$$
\begin{gathered}
G(\boldsymbol{X}, \boldsymbol{Y})=\gamma\left(g_{i}(\boldsymbol{X}, \boldsymbol{Y})+g_{t}(\boldsymbol{X}, \boldsymbol{Y})\right)= \\
\gamma\left(\operatorname{tr}\left(\boldsymbol{X I}^{\mathrm{T}} \boldsymbol{L}_{i} \boldsymbol{I X}^{\mathrm{T}}\right)+\operatorname{tr}\left(\boldsymbol{Y} \boldsymbol{T}^{\mathrm{T}} \boldsymbol{L}_{t} \boldsymbol{T} \boldsymbol{Y}^{\mathrm{T}}\right)\right)
\end{gathered}
$$

\section{3 优化求解}

假设 $\mathrm{I} 2 \mathrm{~T}$ 任务中图片和文本对应的映射矩阵 分别为 $\boldsymbol{X}_{1} \in \mathbb{R}^{c \times d^{i}}$ 和 $\boldsymbol{Y}_{1} \in \mathbb{R}^{c \times d^{t}}$ ，根据式(4)的通用 框架并结合式(5)(6)(8)(11)(18), 可得 I2T 任务的总 目标函数为

$$
\begin{aligned}
& f\left(\boldsymbol{X}_{1}, \boldsymbol{Y}_{1}\right)=\underset{\boldsymbol{X}_{1}, \boldsymbol{Y}_{1}}{\arg \min } \lambda\left\|\boldsymbol{I}_{1}^{\mathrm{T}}-\boldsymbol{T} \boldsymbol{Y}_{1}^{\mathrm{T}}\right\|_{\mathrm{F}}^{2}+ \\
& \\
&(1-\lambda)\left\|\boldsymbol{I}_{1}^{\mathrm{T}}-\boldsymbol{S}\right\|_{\mathrm{F}}^{2}+\alpha_{1}\left\|\boldsymbol{X}_{1}\right\|_{\mathrm{F}}^{2}+\alpha_{2}\left\|\boldsymbol{Y}_{1}\right\|_{\mathrm{F}}^{2}+ \\
& \quad \beta\left(\operatorname{tr}\left(\boldsymbol{Y}_{1} \boldsymbol{M}_{a}^{t} \boldsymbol{Y}_{1}^{\mathrm{T}}\right)-\operatorname{tr}\left(\boldsymbol{Y}_{1} \boldsymbol{M}_{b}^{t} \boldsymbol{Y}_{1}^{\mathrm{T}}\right)\right)+ \\
& \gamma\left(\operatorname{tr}\left(\boldsymbol{X}_{1} \boldsymbol{I}^{\mathrm{T}} \boldsymbol{L}_{i} \boldsymbol{I} \boldsymbol{X}_{1}^{\mathrm{T}}\right)+\operatorname{tr}\left(\boldsymbol{Y}_{1} \boldsymbol{T}^{\mathrm{T}} \boldsymbol{L}_{t} \boldsymbol{T} \boldsymbol{Y}_{1}^{\mathrm{T}}\right)\right)
\end{aligned}
$$

其中, 均衡参数 $\lambda, \beta, \gamma$ 分别表示相关性分析项、 语义回归项、线性判别分析项以及图正则化项的重 要性; $\alpha_{1}$ 和 $\alpha_{2}$ 为正则化参数, 用于平衡映射矩阵 $\boldsymbol{X}_{1}$ 和 $\boldsymbol{Y}_{1}$.

假设 T2I 任务中图片和文本对应的映射矩阵 分别为 $\boldsymbol{X}_{2} \in \mathbb{R}^{c \times d^{i}}$ 和 $\boldsymbol{Y}_{2} \in \mathbb{R}^{c \times d^{t}}$ ，根据式(4)并结合 式(5)(7)(8)(14)(18), 可得 T2I 任务的总目标函数为

$$
\begin{gathered}
f\left(\boldsymbol{X}_{2}, \boldsymbol{Y}_{2}\right)=\underset{\boldsymbol{X}_{2}, \boldsymbol{Y}_{2}}{\arg \min } \lambda\left\|\boldsymbol{I}_{2}{ }^{\mathrm{T}}-\boldsymbol{T} \boldsymbol{Y}_{2}^{\mathrm{T}}\right\|_{\mathrm{F}}^{2}+ \\
(1-\lambda)\left\|\boldsymbol{T} \boldsymbol{Y}_{2}^{\mathrm{T}}-\boldsymbol{S}\right\|_{\mathrm{F}}^{2}+\alpha_{1}\left\|\boldsymbol{X}_{2}\right\|_{\mathrm{F}}^{2}+\alpha_{2}\left\|\boldsymbol{Y}_{2}\right\|_{\mathrm{F}}^{2}+ \\
\beta\left(\operatorname{tr}\left(\boldsymbol{X}_{2} \boldsymbol{M}_{a}^{i} \boldsymbol{X}_{2}^{\mathrm{T}}\right)-\operatorname{tr}\left(\boldsymbol{X}_{2} M_{b}^{i} \boldsymbol{X}_{2}^{\mathrm{T}}\right)\right)+ \\
\gamma\left(\operatorname{tr}\left(\boldsymbol{X}_{2} \boldsymbol{I}^{\mathrm{T}} \boldsymbol{L}_{i} \boldsymbol{I} \boldsymbol{X}_{2}^{\mathrm{T}}\right)+\operatorname{tr}\left(\boldsymbol{Y}_{2} \boldsymbol{T}^{\mathrm{T}} \boldsymbol{L}_{t} \boldsymbol{T} \boldsymbol{Y}_{2}^{\mathrm{T}}\right)\right)
\end{gathered}
$$


I2T 和 T2I 任务的优化求解问题为包含 2 个矩阵变 量的无约束优化. 因此, 式(19)(20)均为非凸优化 问题，且有一些局部最优解. 对于此类非凸问题， 通常需设计方法来寻找其鞍点. 对式(19)(20)中需 求解的映射矩阵 $\boldsymbol{X}$ 和 $\boldsymbol{Y}$, 当固定 $\boldsymbol{X}(\boldsymbol{Y})$ 时, 对 $\boldsymbol{Y}(\boldsymbol{X})$ 的求解将变成一个凸问题. 以 $\mathrm{I} 2 \mathrm{~T}$ 任务为 例, 固定 $\boldsymbol{Y}_{1}, f\left(\boldsymbol{X}_{1}, \boldsymbol{Y}_{1}\right)$ 对 $\boldsymbol{X}_{1}$ 求偏导并置零, 可得

$$
\begin{aligned}
& \frac{\partial f\left(\boldsymbol{X}_{1}, \boldsymbol{Y}_{1}\right)}{\partial \boldsymbol{X}_{1}}=\lambda\left(\boldsymbol{X}_{1} \boldsymbol{I}^{\mathrm{T}} \boldsymbol{I}-\boldsymbol{Y}_{1} \boldsymbol{T}^{\mathrm{T}} \boldsymbol{I}\right)+ \\
& (1-\lambda)\left(\boldsymbol{X}_{1} \boldsymbol{I}^{\mathrm{T}} \boldsymbol{I}-\boldsymbol{S}^{\mathrm{T}} \boldsymbol{I}\right)+\alpha_{1} \boldsymbol{X}_{1}+\gamma \boldsymbol{X}_{1} \boldsymbol{I}^{\mathrm{T}} \boldsymbol{L}_{i} \boldsymbol{I} .
\end{aligned}
$$

那么

$$
\begin{gathered}
\boldsymbol{X}_{1}=\left(\lambda \boldsymbol{Y}_{1} \boldsymbol{T}^{\mathrm{T}} \boldsymbol{I}+(1-\lambda) \boldsymbol{S}^{\mathrm{T}} \boldsymbol{I}\right) \times \\
\left(\boldsymbol{I}^{\mathrm{T}} \boldsymbol{I}+\alpha_{1} \boldsymbol{E}+\gamma \boldsymbol{I}^{\mathrm{T}} \boldsymbol{L}_{i} \boldsymbol{I}\right)^{-1}
\end{gathered}
$$

固定 $\boldsymbol{X}_{1}, f\left(\boldsymbol{X}_{1}, \boldsymbol{Y}_{1}\right)$ 对 $\boldsymbol{Y}_{1}$ 求偏导并置零, 可得到

$$
\begin{gathered}
\frac{\partial f\left(\boldsymbol{X}_{1}, \boldsymbol{Y}_{1}\right)}{\partial \boldsymbol{Y}_{1}}=\lambda\left(\boldsymbol{Y}_{1} \boldsymbol{T}^{\mathrm{T}} \boldsymbol{T}-\boldsymbol{X}_{1} \boldsymbol{I}^{\mathrm{T}} \boldsymbol{T}\right)+\alpha_{2} \boldsymbol{Y}_{1}+ \\
\beta \boldsymbol{Y}_{1}\left(\boldsymbol{M}_{a}^{t}\right)^{\mathrm{T}}-\beta \boldsymbol{Y}_{1}\left(\boldsymbol{M}_{b}^{t}\right)^{\mathrm{T}}+\gamma \boldsymbol{Y}_{1} \boldsymbol{T}^{\mathrm{T}} \boldsymbol{L}_{t} \boldsymbol{T} . \\
\boldsymbol{Y}_{1}=\lambda\left(\boldsymbol{X}_{1} \boldsymbol{I}^{\mathrm{T}} \boldsymbol{T}\right)\left(\lambda \boldsymbol{T}^{\mathrm{T}} \boldsymbol{T}+\alpha_{2} \boldsymbol{E}+\beta\left(\boldsymbol{M}_{a}^{t}\right)^{\mathrm{T}}-\right. \\
\left.\beta\left(\boldsymbol{M}_{b}^{t}\right)^{\mathrm{T}}+\gamma \boldsymbol{T}^{\mathrm{T}} \boldsymbol{L}_{t} \boldsymbol{T}\right)^{-1}
\end{gathered}
$$

同样, T2I 任务中习得的映射矩阵 $\boldsymbol{X}_{2}$ 和 $\boldsymbol{Y}_{2}$ 为

$$
\begin{gathered}
\boldsymbol{X}_{2}=\lambda\left(\boldsymbol{Y}_{2} \boldsymbol{T}^{\mathrm{T}} \boldsymbol{I}\right)\left(\lambda \boldsymbol{I}^{\mathrm{T}} \boldsymbol{I}+\alpha_{1} \boldsymbol{E}+\beta\left(\boldsymbol{M}_{a}^{i}\right)^{\mathrm{T}}-\right. \\
\left.\beta\left(\boldsymbol{M}_{b}^{i}\right)^{\mathrm{T}}+\gamma \boldsymbol{I}^{\mathrm{T}} \boldsymbol{L}_{i} \boldsymbol{I}\right)^{-1} \\
\boldsymbol{Y}_{2}=\left(\lambda \boldsymbol{X}_{2} \boldsymbol{I}^{\mathrm{T}} \boldsymbol{T}+(1-\lambda) \boldsymbol{S}^{\mathrm{T}} \boldsymbol{T}\right) \times \\
\left(\boldsymbol{T}^{\mathrm{T}} \boldsymbol{T}+\alpha_{2} \boldsymbol{E}+\gamma \boldsymbol{T}^{\mathrm{T}} \boldsymbol{L}_{t} \boldsymbol{T}\right)^{-1}
\end{gathered}
$$

以 $\mathrm{I} 2 \mathrm{~T}$ 任务为例，通过交替迭代算法进行优化 求解，整体算法步骤如下:

输人. 已标记图片矩阵 $\boldsymbol{I}=\left[\boldsymbol{i}_{1}, \boldsymbol{i}_{2}, \cdots, \boldsymbol{i}_{n}\right] \in \mathbb{R}^{n \times d^{i}}$, 已标记文本矩阵 $\boldsymbol{T}=\left[\boldsymbol{t}_{1}, \boldsymbol{t}_{2}, \cdots, \boldsymbol{t}_{n}\right] \in \mathbb{R}^{n \times d^{t}}$, 所有图片 矩阵(含未标记图片) $\boldsymbol{I}^{*}=\left[\boldsymbol{i}_{1}, \boldsymbol{i}_{2}, \cdots, \boldsymbol{i}_{n+m}\right] \in \mathbb{R}^{(n+m) \times d^{i}}$, 所有文本矩阵 $\boldsymbol{T}^{*}=\left[\boldsymbol{t}_{1}, \boldsymbol{t}_{2}, \cdots, \boldsymbol{t}_{n+m}\right] \in \mathbb{R}^{(n+m) \times d^{t}}$, 标签 矩阵 $\boldsymbol{S}=\left\{\boldsymbol{s}_{1}, \boldsymbol{s}_{2}, \cdots, \boldsymbol{s}_{n}\right\} \in \mathbb{R}^{n \times c}$, 均衡参数 $\lambda, \alpha_{1}, \alpha_{2}$, $\beta, \gamma$.

输出. 图片投影矩阵 $\boldsymbol{X}_{1} \in \mathbb{R}^{c \times d^{i}}$ 和文本投影矩 阵 $\boldsymbol{Y}_{1} \in \mathbb{R}^{c \times d^{t}}$.

Step1. 根据式(9)(10)计算文本类内散度矩阵 $\boldsymbol{M}_{a}^{t}$ 和 文本类间散度矩阵 $\boldsymbol{M}_{b}^{t}$.
Step2. 根据式(16)(17)计算图片模态图的拉普拉斯 矩阵 $\boldsymbol{L}_{i} \in \mathbb{R}^{(n+m) \times(n+m)}$ 和文本模态图的拉普拉斯矩阵 $\boldsymbol{L}_{t} \in \mathbb{R}^{(n+m) \times(n+m)}$.

Step3. 令 $l=0$, 并初始化投影矩阵 $\boldsymbol{X}_{1}^{(0)}, \boldsymbol{Y}_{1}^{(0)}$ 为零 矩阵.

Step4. 重复以下步骤直到收敛:

Step4.1. 根据式(21)更新 $\boldsymbol{X}_{1}^{(l)}$, 那么 $\boldsymbol{X}_{1}^{(l)}$ 的迭 代规则为

$$
\boldsymbol{X}_{1}^{(l+1)}=\left(\lambda \boldsymbol{Y}_{1} \boldsymbol{T}^{\mathrm{T}} \boldsymbol{I}+(1-\lambda) \boldsymbol{S}^{\mathrm{T}} \boldsymbol{I}\right)\left(\boldsymbol{I}^{\mathrm{T}} \boldsymbol{I}+\alpha_{1} \boldsymbol{E}+\gamma \boldsymbol{I}^{\mathrm{T}} \boldsymbol{L}_{i} \boldsymbol{I}\right)^{-1} ;
$$

Step4.2. 根据式(22)更新 $\boldsymbol{Y}_{1}^{(l)}$ ，那么 $\boldsymbol{Y}_{1}^{(l)}$ 的迭代 规则为

$$
\begin{gathered}
\boldsymbol{Y}_{1}^{(l+1)}=\lambda\left(\boldsymbol{X}_{1}^{(l)} \boldsymbol{I}^{\mathrm{T}} \boldsymbol{T}\right)\left(\lambda \boldsymbol{T}^{\mathrm{T}} \boldsymbol{T}+\alpha_{2} \boldsymbol{E}+\beta\left(\boldsymbol{M}_{a}^{t}\right)^{\mathrm{T}}-\right. \\
\left.\beta\left(\boldsymbol{M}_{b}^{t}\right)^{\mathrm{T}}+\gamma \boldsymbol{T}^{\mathrm{T}} \boldsymbol{L} \boldsymbol{T}\right)^{-1} ;
\end{gathered}
$$

Step4.3. $l=l+1$

\section{3 实 验}

\section{1 实验数据集}

在广泛使用的公开的跨模态数据集 Wikipedia 和 Pascal Sentence 上, 对本文方法进行评估.

(1) Wikipedia 数据集 ${ }^{[6]}$ 由维基百科的“特色文 章”中 10 个数量最多的语义类别组成, 如生物和地 理等，总共有 2866 个图片文本对. 整个数据集被 随机划分为 2 部分, 其中训练集包含 2173 个图片 文本对，其余 693 个为测试集. 本实验使用文献 [11]提供的公开特征, 其中图片特征为 4096 维卷 积神经网络(convolutional neural networks, CNN)特 征, 文本特征为 100 维隐含狄利克雷分布(latent Dirichlet alloction, LDA)特征.

(2) Pascal Sentence 数据集 ${ }^{[19]}$ 由 2008 Pascal 开 发工具包中提取的 1000 个图片文本对组成, 其中 每张图片对应的文本由 5 句人工标注的独立语句 构成. 整个数据集可分为 20 类，每个类别有 50 个 图片文本对, 从每类样本中随机选择 30 个图片文 本对作为训练集, 其余为测试集. 与 Wikipedia 数 据集一样, 图片特征为 4096 维 $\mathrm{CNN}$ 特征, 文本特 征为 100 维 $\mathrm{LDA}$ 特征.

\section{2 评估指标和对比方法}

为了评估本文方法的性能, 在上述数据集上 进行了 $\mathrm{I} 2 \mathrm{~T}$ 和 T2I 这 2 个跨模态检索任务. 以 $\mathrm{I} 2 \mathrm{~T}$ 任务为例, 给定测试集中的 1 张图片作为查询样 本，检索测试集中与其相关的文本，度量其相似度 并进行排序. 


\subsection{1 评估指标}

本文使用归一化相关系数(normalized correlation，NC)来度量公共子空间中不同模态样本间的相 似性，并采用平均精度均值(mean average precision, mAP)作为评价指标, mAP 值越大, 方法性能越好. 此外，本文中的 mAP 值由每个样例返回的所有的 检索结果计算得到. 每次实验时，先根据单个查询 样本的排序结果得到其相应的平均准确率(average precision, AP), 再对所有查询样本的 AP 值进行平 均, 即可得到最终的 mAP 值. AP 值的计算公式为

$$
\mathrm{AP}=\frac{1}{L} \sum_{i=1}^{r} p(i) \times \delta(i) .
$$

其中， $L$ 表示检索到的样本总数; $r$ 表示检索到的 与查询样本相关的样本数量; $p(i)$ 为检索到相关 样本 $i$ 的准确率; $\delta(i)$ 为指示函数， $\delta(i)=1$ 表示检 索到的第 $i$ 个样本与查询样本相关, 否则 $\delta(i)=0$.

\section{2 .2 对比方法}

为了显示本文方法的可行性，与 12 种现有的 跨模态检索方法进行了比较, 包括 $\mathrm{PLS}^{[4]}, \mathrm{CCA}^{[2]}$, 语义匹配法(semantic match, SM) ${ }^{[6]}$ 、语义关联匹配 法(semantic correlation match, SCM) ${ }^{[6]}$ 、广义多视角 线性判别分析法 (generalized multiview linear dicriminant analysis, GMLDA) ${ }^{[20]}$ 、广义多视图边缘 Fisher 分析法(generalized multiview marginal fisher analysis, GMMFA) ${ }^{[20]}$ 、联合特征选择的子空间学习 (joint feature selection and subspce learning, JFSSL) ${ }^{[21]}$ 、联合子空间学习和回归学习(joint latent

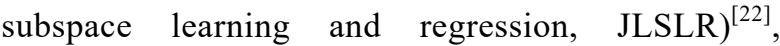
$\mathrm{CRLDA}^{[10]}, \mathrm{MDCR}^{[11]}, \mathrm{MJSL}^{[12]}$ 、基于中层语义增强 空间的跨模态检索法 (modal-dependent retrieval based on mid-level semantic enhancement space, MMSES $)^{[14]}$. 其中, 后 3 种方法为不同的检索任务 学习不同的映射矩阵. 此外, 为了公平比较, 实验 中所有对比方法与本文方法使用的数据集和样本 特征均相同。

\section{3 实验结果与分析}

\subsection{1 消融实验}

为了验证本文方法各部分的效果，设计了针 对性的消融实验，其中“无线性判别”表示去掉总 目标函数(见式(19)(20))中的线性判别分析项(见式 (11)(14)), “无图正则化”表示去掉总目标函数中的 图正则化项(见式(18)), “非任务导向”表示将目标 函数中的单模态语义回归项(见式(6)(7)) 合并为多 模态语义回归、单模态线性判别分析项合并为多模 态线性判别分析项，即对不同检索任务采用相同 的目标函数.
从表 1 的实验结果可知, 通过线性判别分析对 检索模态的样本进行类内聚合、类间远离，可以有 效地保证其语义一致性. 该项在图片检索文本任 务中表现更佳，这是因为文本特征的判别性更强， 可以保留更多的语义信息. 为不同模态的数据构 建 $k \mathrm{NN}$ 局部近邻图, 可以更好地保留模态内的相 似性. 而忽略不同任务的差异性，使用多模态语义 回归和多模态线性判别分析，即对不同任务使用 相同的目标函数，只学习一组映射反而使得每个 任务中的性能无法达到各自最优. 此外, 线性判别 分析项和图正则化项可以互相促进，进一步提高 跨模态检索的准确率.

表 1 本文方法各部分在 2 个数据集上 mAP 对比

\begin{tabular}{lcccccccc}
\hline \multirow{2}{*}{ 方法 } & \multicolumn{3}{c}{ Wikipedia } & & \multicolumn{3}{c}{ Pascal Sentence } \\
\cline { 2 - 3 } \cline { 7 - 9 } & I2T & T2I & 平均 & & I2T & T2I & 平均 \\
\hline 无线性判别 & 0.435 & 0.405 & 0.420 & & 0.490 & 0.500 & 0.495 \\
无图正则化 & 0.434 & 0.392 & 0.413 & & 0.488 & 0.495 & 0.492 \\
非任务导向 & 0.403 & 0.346 & 0.375 & & 0.477 & 0.485 & 0.481 \\
本文 & 0.447 & 0.407 & 0.427 & 0.494 & 0.505 & 0.500 \\
\hline
\end{tabular}

\subsection{2 与现有方法进行对比}

本文方法和现有方法在 Wikipedia 数据集和 Pascal Sentence 数据集上的对比结果如表 2 所示. 由表 2 可知, $\mathrm{I} 2 \mathrm{~T}$ 任务中本文方法在 2 个数据集上 的 $\mathrm{mAP}$ 分别为 0.447 和 0.494 , T2I 任务中本文方法 在 2 个数据集上的 $\mathrm{mAP}$ 值分别为 0.407 和 0.505 , 各检索任务的性能都有一定提升，在 2 个数据集上 均超过了 12 种对比方法.

在对比方法中, CCA 和 PLS 只考虑了多模态

表 2 不同方法在 2 个数据集上 mAP 对比

\begin{tabular}{lccccccc}
\hline \multirow{2}{*}{ 方法 } & \multicolumn{3}{c}{ Wikipedia } & & \multicolumn{3}{c}{ Pascal Sentence } \\
\cline { 2 - 3 } \cline { 7 - 8 } & I2T & T2I & 平均 & & I2T & T2I & 平均 \\
\hline PLS $^{[4]}$ & 0.360 & 0.351 & 0.355 & & 0.365 & 0.376 & 0.371 \\
CCA $^{[2]}$ & 0.332 & 0.317 & 0.325 & & 0.380 & 0.372 & 0.376 \\
SM$^{[6]}$ & 0.369 & 0.387 & 0.378 & & 0.450 & 0.434 & 0.442 \\
SCM $^{[6]}$ & 0.375 & 0.393 & 0.384 & & 0.407 & 0.394 & 0.400 \\
GMMFA $^{[20]}$ & 0.284 & 0.249 & 0.267 & & 0.373 & 0.347 & 0.360 \\
GMLDA $^{[20]}$ & 0.300 & 0.281 & 0.291 & & 0.408 & 0.388 & 0.398 \\
JFSSL $^{[21]}$ & 0.392 & 0.381 & 0.387 & & 0.407 & 0.402 & 0.405 \\
MDCR $^{[11]}$ & 0.411 & 0.378 & 0.395 & & 0.432 & 0.462 & 0.447 \\
JLSLR $^{[22]}$ & 0.394 & 0.369 & 0.382 & & 0.454 & 0.455 & 0.455 \\
MJSL $^{[12]}$ & 0.443 & 0.383 & 0.413 & & 0.487 & 0.461 & 0.474 \\
CRLDA $^{[10]}$ & 0.429 & 0.399 & 0.414 & & 0.478 & 0.488 & 0.483 \\
MMSES $^{[14]}$ & 0.438 & 0.395 & 0.417 & & 0.485 & 0.490 & 0.488 \\
本文 & $\mathbf{0 . 4 4 7}$ & $\mathbf{0 . 4 0 7}$ & $\mathbf{0 . 4 2 7}$ & & $\mathbf{0 . 4 9 4}$ & $\mathbf{0 . 5 0 5}$ & $\mathbf{0 . 5 0 0}$ \\
\hline
\end{tabular}

注. 粗体表示最优实验结果. 
数据间的成对相关性，在 2 个数据集上的表现较 差, JFSSL 虽在一个耦合框架中根据 $k$ 近邻和标签 信息为多模态数据构建了模态内和模态间的图模 型，但其忽略了不同检索任务的差异性，在 2 个数 据集上的表现都不如 MDCR，MJSL，MMSES 这 3 种方法. 而本文方法表现较优的主要原因如下：一 方面，本文方法既考虑了不同检索任务的差异性 利用单模态语义回归增强查询模态的语义准确性, 又利用线性判别分析在一定程度上保留了检索模 态的语义一致性；另一方面，本文方法在同一框架 中结合了样本的局部近邻关系，可以挖掘数据间 更多的匹配信息, 从而能进一步提升检索性能. Wikipedia 数据集和 Pascal Sentence 数据集上的查 准率-查全率曲线分别如图 3 和图 4 所示.

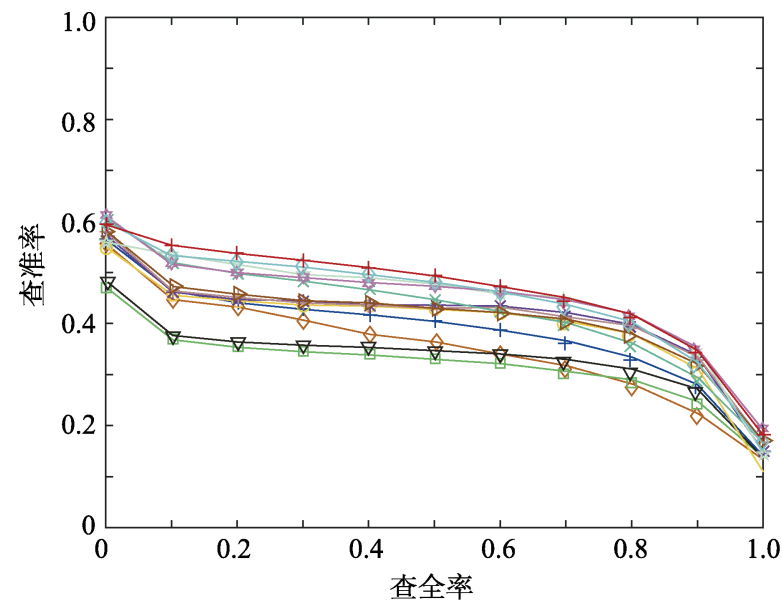

a. I2T任务的查准率-查全率曲线

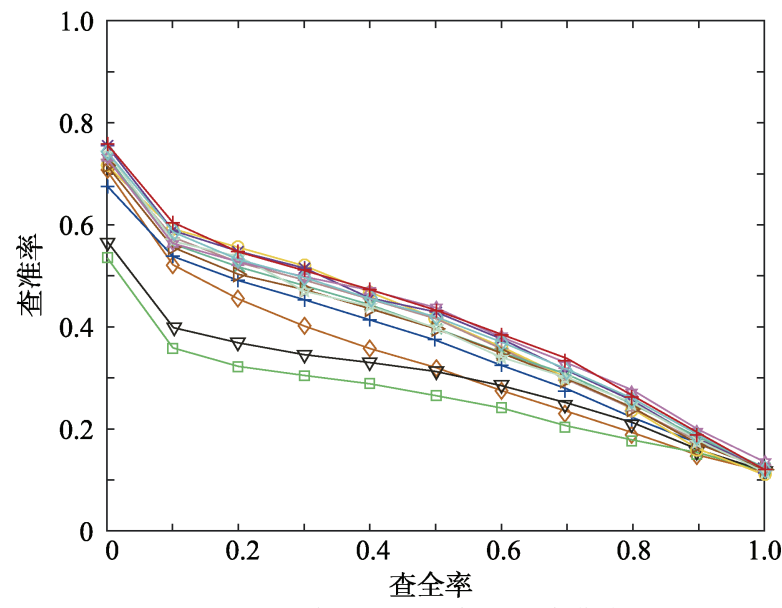

b. T2I任务的查准率-查全率曲线

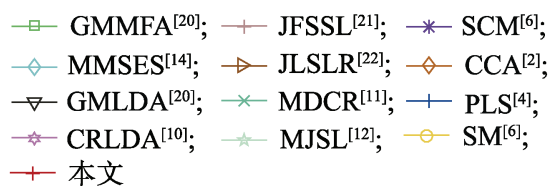

图 3 Wikipedia 数据集的查准率-查全率曲线

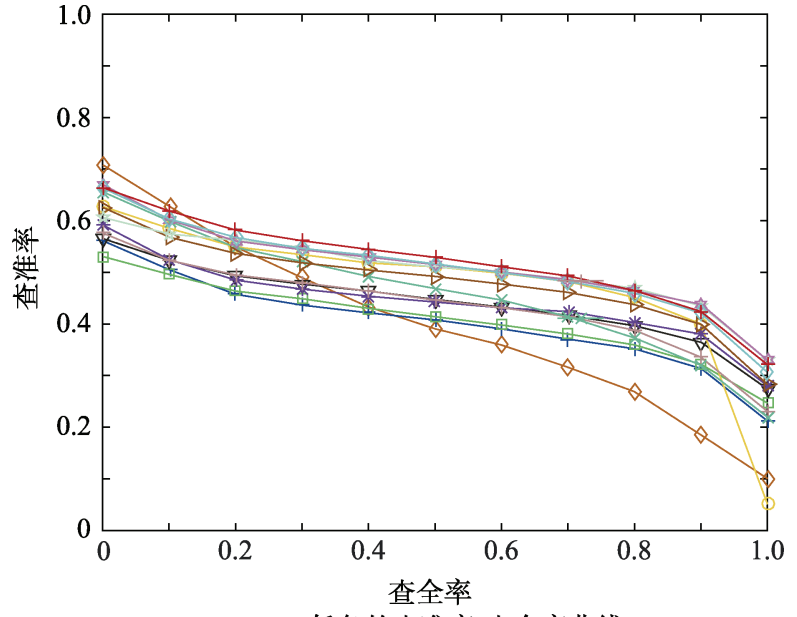

a. I2T 任务的查准率-查全率曲线

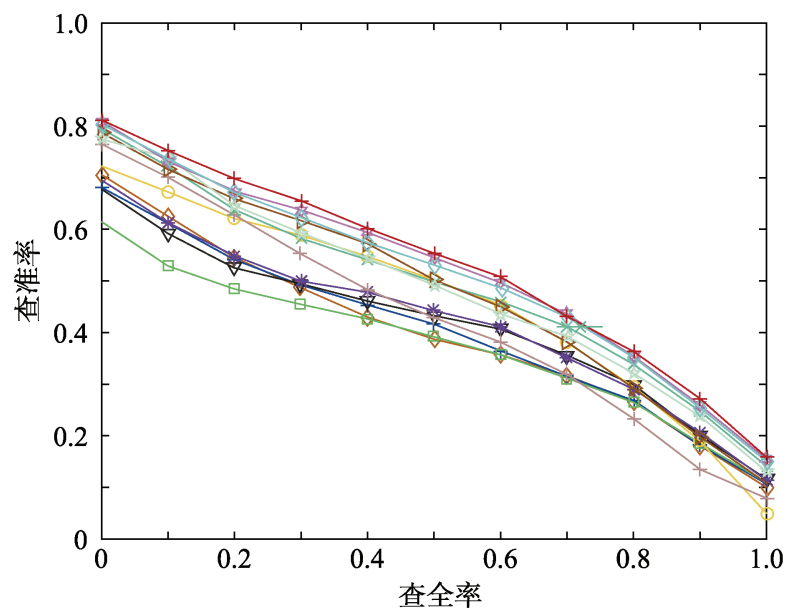

b. T2I 任务的查准率-查全率曲线

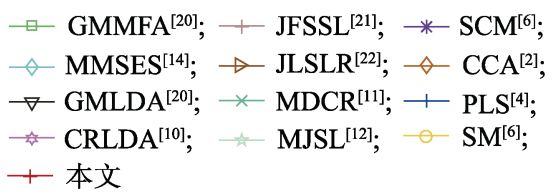

图 4 Pascal Sentence 数据集的查准率-查全率曲线

\subsection{3 实验效率和收敛性分析}

为进一步探讨本文方法的可行性，进行了效 率和收玫性分析. 本实验基于 Matlab 2016 平台实 现, 在 2 个数据集上的训练时间如表 3 所示. 在目 标函数的线性判别分析项中, 图片和文本模态的 类内散度矩阵与类间散度矩阵以及图正则化项中 图片和文本模态的拉普拉斯矩阵都采用离线保存 读取的方式.

表 3 本文方法各部分在 2 个数据集上的训练时间对比 $\mathrm{s}$

\begin{tabular}{lccccccc}
\hline \multirow{2}{*}{ 方法 } & \multicolumn{3}{c}{ Wikipedia } & & \multicolumn{3}{c}{ Pascal Sentence } \\
\cline { 2 - 3 } \cline { 7 - 8 } & I2T & T2I & 平均 & & I2T & T2I & 平均 \\
\hline 无线性判别 & 57.038 & 61.848 & 59.443 & & 23.821 & 24.922 & 24.372 \\
无图正则化 & 46.010 & 47.035 & 46.523 & & 22.645 & 23.551 & 23.098 \\
非任务导向 & 57.427 & 57.427 & 57.427 & & 23.987 & 23.987 & 23.987 \\
本文 & 57.237 & 61.615 & 59.426 & & 23.874 & 18.834 & 21.354 \\
\hline
\end{tabular}


由表 3 可知, 目标函数的不同项在 2 个数据集 上对训练时间影响不同. 在 Wikipedia 数据集中, 图正则化项对训练时间影响较大，线性判别分析 项对训练时间几乎无影响; 而在 Pascal Sentence 数 据集中，加人图正则化或线性判别分析均能减少 训练时间. 其主要原因在于, Wikipedia数据集的语 义类别十分抽象，同模态数据类别差异不明显，导 致增加图正则化后收玫变慢, 而 Pascal Sentence数 据集类别差异明显，增加这 2 项反而有助于收敛. 此外，对比非任务导向和本文方法的训练时间，可 知任务导向方法中不同检索任务的收敛速度不同， 但平均训练时间与非任务导向方法相比差异不大.

在 2 个数据集上绘制的收敛曲线分别如图 5 和图 6 所示. 结果显示本文方法的目标函数值随着 迭代次数的增加逐渐稳定，且一般在 5 次迭代后即 可收敛.

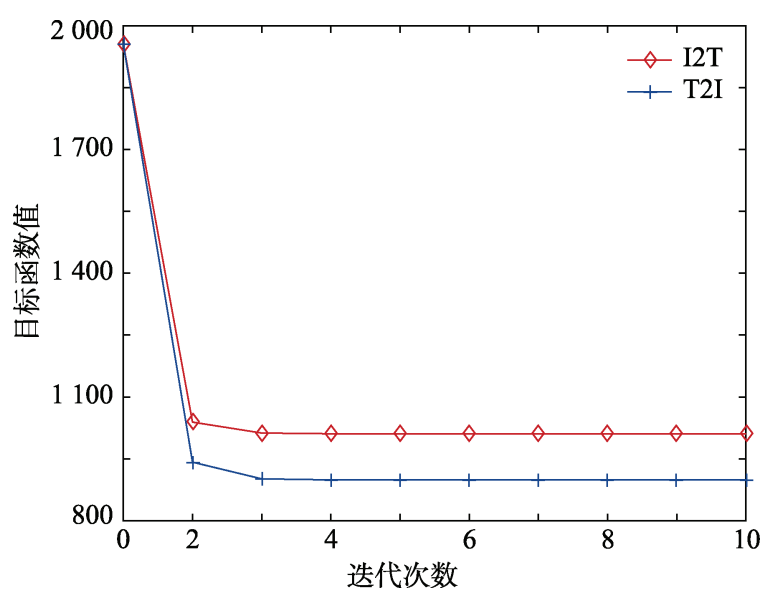

图 5 Wikipedia 数据集的收敛曲线

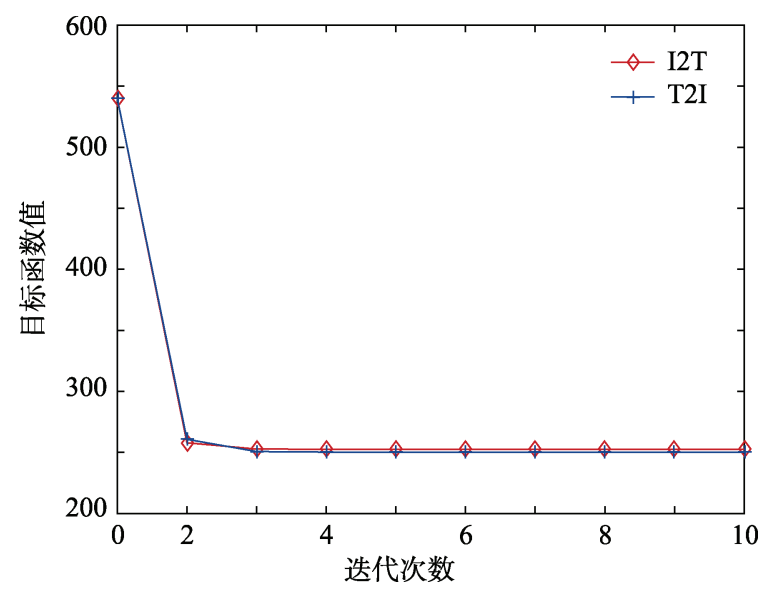

图 6 Pascal Sentence 数据集的收敛曲线

\section{4 结 语}

本文提出一种联合线性判别和图正则的任务
导向型跨模态检索方法，对不同的检索任务采用 不同的映射机制. 联合不同模态成对样本间的相 关性和查询模态样本特征向其对应的语义向量的 回归将多模态数据映射到公共子空间，同时在联 合学习过程中, 利用检索模态的线性判别分析项 充分保证检索模态样本的语义一致性, 并为每个 模态构建局部近邻图来挖掘其模态内的原始分布 信息, 从而得到更多的数据匹配信息. 在 2 个跨模 态数据集上与 12 种现有方法对比, 实验结果充分 显示了本文方法的有效性. 此外, 本文方法目标函 数中的各项都不局限于某种特定模态, 易于拓展 到除图片文本外的其他模态, 且可不限模态叠加, 因而其理论上可在同一框架中实现 2 种模态以上的 检索. 未来可尝试结合深度学习与跨模态数据的细 粒度信息，以实现更多模态数据间的精确检索.

\section{参考文献(References):}

[1] Peng Yuxin, Qi Jinwei, Huang Xin. Current research status and prospects on multimedia content understanding[J]. Journal of Computer Research and Development, 2019, 56(1): 183-208(in Chinese)

(彭宇新, 綦金玮, 黄金金. 多媒体内容理解的研究现状与展 望[J]. 计算机研究与发展, 2019, 56(1): 183-208)

[2] Hotelling H. Relations between two sets of variates[J]. Biometrika, 1936, 28(3/4): 321-377

[3] Hardoon D R, Szedmak S, Shawe-taylor J R. Canonical correlation analysis: an overview with application to learning methods[J]. Neural Computation, 2004, 16(12): 2639-2664

[4] Rosipal R, Krämer N. Overview and recent advances in partial least squares[C] //Proceedings of International Conference on Subspace, Latent Structure and Feature Selection. Heidelberg: Springer, 2005: 34-51

[5] Li D G, Dimitrova N, Li M K, et al. Multimedia content processing through cross-modal association[C] //Proceedings of 11th ACM International Conference on Multimedia. New York: ACM Press, 2003: 604-611

[6] Rasiwasia N, Costa Pereira J, Coviello E, et al. A new approach to cross-modal multimedia retrieval[C] //Proceedings of the 18th ACM International Conference on Multimedia. New York: ACM Press, 2010: 251-260

[7] Gong Y C, Ke Q F, Isard M, et al. A multi-view embedding space for modeling internet images, tags, and their semantics[J]. International Journal of Computer Vision, 2014, 106(2): 210-233

[8] Zhai X H, Peng Y X, Xiao J G. Learning cross-media joint representation with sparse and semisupervised regularization[J]. IEEE Transactions on Circuits and Systems for Video Technology, 2014, 24(6): 965-978

[9] Zhang L, Ma B P, Li G R, et al. Generalized semi-supervised and structured subspace learning for cross-modal retrieval[J]. IEEE Transactions on Multimedia, 2018, 20(1): 128-141 
[10] Qi Y D, Zhang H X, Zhang B, et al. Cross-media retrieval based on linear discriminant analysis[J]. Multimedia Tools and Applications, 2019, 78(17): 24249-24268

[11] Wei Y C, Zhao Y, Zhu Z F, et al. Modality-dependent cross-media retrieval[J]. ACM Transactions on Intelligent Systems and Technology, 2016, 7(4): Article No.57

[12] Yu E, Li J, Wang L, et al. Multi-class joint subspace learning for cross-modal retrieval[J]. Pattern Recognition Letters, 2020, 130: $165-173$

[13] Yan J H, Zhang H X, Sun J D, et al. Joint graph regularization based modality-dependent cross-media retrieval[J]. Multimedia Tools and Applications, 2018, 77(3): 3009-3027

[14] Zheng S X, Zhang H X, Qi Y D, et al. Modal-dependent retrieval based on mid-level semantic enhancement space[J]. IEEE Access, 2019, 7: 49906-49917

[15] Kettenring J R. Canonical analysis of several sets of variables[J]. Biometrika, 1971, 58(3): 433-451

[16] Li H F, Jiang T, Zhang K S. Efficient and robust feature extraction by maximum margin criterion[J]. IEEE Transactions on Neural Networks, 2006, 17(1): 157-165

[17] Peng Y X, Zhai X H, Zhao Y Z, et al. Semi-supervised cross-media feature learning with unified patch graph regularization[J]. IEEE Transactions on Circuits and Systems for Video
Technology, 2016, 26(3): 583-596

[18] Yao T, Kong X W, Fu H Y, et al. Semantic consistency hashing for cross-modal retrieval[J]. Neurocomputing, 2016, 193: 250-259

[19] Rashtchian C, Young P, Hodosh M, et al. Collecting image annotations using Amazon's Mechanical Turk[C] //Proceedings of the Workshop on Creating Speech and Language Data with Amazon's Mechanical Turk. Stroudsburg: ACL Press, 2010: 139-147

[20] Sharma A, Kumar A, Daume H, et al. Generalized multiview analysis: a discriminative latent space[C] //Proceedings of the IEEE Conference on Computer Vision and Pattern Recognition. Los Alamitos: IEEE Computer Society Press, 2012: 2160-2167

[21] Wang K Y, He R, Wang L, et al. Joint feature selection and subspace learning for cross-modal retrieval[J]. IEEE Transactions on Pattern Analysis and Machine Intelligence, 2016, 38(10): 2010-2023

[22] Wu J L, Lin Z C, Zha H B. Joint latent subspace learning and regression for cross-modal retrieval[C] //Proceedings of the 40th International ACM SIGIR Conference on Research and Development in Information Retrieval. New York: ACM Press, 2017: 917-920 